\title{
CRESCIMENTO DE TRÊS CLONES DE UMEZEIRO (Prunus mume Sieb. et Zucc.) E PESSEGUEIRO CV. OKINAWA (Prunus persica (L.) Batsch.) PROPAGADOS POR ESTACAS HERBÁCEAS ${ }^{1}$
}

\author{
NEWTON ALEX MAYER ${ }^{2} \&$ FERNANDO MENDES PEREIRA ${ }^{3}$
}

\begin{abstract}
RESUMO - O umezeiro (Prunus mume Sieb. et Zucc.) apresenta promissoras características para utilização como porta-enxerto para pessegueiro e nectarineira; entretanto, quando adotada a propagação sexuada, as plantas apresentam-se muito heterogêneas, em função da grande variabilidade genética da espécie. Três clones (Clones 05; 10 e 15), selecionados a partir de plantas em cultivo na Estação Experimental de Jundiaí-SP (IAC), propagados assexuadamente e mantidos na FCAV/UNESP, Câmpus de Jaboticabal-SP, têm sido objeto de diversos estudos para viabilizar agronomicamente estes materiais. O presente estudo teve por objetivo avaliar o crescimento de três clones de umezeiro e do pessegueiro cv. Okinawa propagados por estacas herbáceas. As estacas enraizadas foram transplantadas para sacolas plásticas, mantidas em condição de viveiro telado, e avaliadas (diâmetro e comprimento da haste) até 145 dias. Os Clones 05; 10 e 15 de umezeiro apresentam crescimento contínuo com relação ao comprimento da haste, enquanto a cv. Okinawa estabiliza o crescimento após 100 dias do transplantio, refletindo-se em plantas mais baixas. Não existem diferenças entre os clones de umezeiro estudados no diâmetro da haste após 100 dias e no comprimento da haste após 115 dias do transplantio das estacas enraizadas. A cv. Okinawa apresenta maior diâmetro da haste em relação aos Clones 05; 10 e 15 de umezeiro, o que define maior porcentagem de porta-enxertos aptos à realização da enxertia.
\end{abstract}

Termos para indexação: Porta-enxerto, propagação vegetativa, Prunus spp.

\section{GROWTH OF THREE MUME CLONES (Prunus mume Sieb. et Zucc.) AND 'OKINAWA’ PEACH (Prunus persica (L.) Batsch.) PROPAGATED BY HERBACEOUS CUTTINGS}

\begin{abstract}
Mume (Prunus mume Sieb. et Zucc.) presents promising characteristics for use as rootstock for peach and nectarines trees, however, when adopted the sexual propagation, it results in very heterogeneous plants, as a function of the mume's great genetic variability. Three clones (Clones 05, 10 and 15), selected from plants grown in the Estação Experimental de Jundiaí (IAC), São Paulo State, Brazil, propagated vegetatively and maintained in FCAV/UNESP, Jaboticabal Campus, have been object of several studies to make possible the agronomical use of these materials. The present study had for objective to evaluate the growth of three mume clones and 'Okinawa' peach propagated by herbaceous cuttings. The rooted cuttings were transplanted for plastic bags, maintained in nursery conditions and evaluated (stem diameter and length) up to 145 days. The mume Clones 05, 10 and 15 presented continuous growth with relationship to stem length, while 'Okinawa' peach stabilized growth 100 days after transplant, reflecting in lower plants. Differences don't exist among the mume clones studied in stem diameter after 100 days and stem length after 115 days of rooted cuttings transplant. 'Okinawa' peach presents larger stem diameter in relation to mume Clones 05,10 and 15 , what defines larger percentage of capable rootstocks for accomplishment of graft.
\end{abstract}

Index terms: Rootstock, vegetative propagation, Prunus spp.

\section{INTRODUÇÃO}

O umezeiro (Prunus mume Sieb. et Zucc.) é uma Rosaceae arbórea, de folhas caducas e nativa da China. No Japão, as primeiras cultivares foram introduzidas há 2.000 anos e adquiriram significativa expressão na alimentação e nos costumes orientais. No ano de 1991, eram cultivadas no Japão cerca de 300 cultivares de umezeiro, que ocupavam uma área de 18.900 hectares e produção de 94.500 toneladas. Os japoneses também cultivam o umezeiro em jardins, em forma de bonsai, e utilizam as flores no preparo de arranjos. Os frutos, geralmente ácidos, podem ser consumidos na forma de picles, licores especiais (ume-shu), conservas (ume-boshi), compotas, geléias, sucos, extratos, na confecção de bolos e como uso medicinal, sendo associados a uma vida saudável (Yoshida, 1994).

A introdução desta espécie no Brasil deu-se, provavelmente, através dos imigrantes japoneses, que obtiveram produções satisfatórias somente a partir de 1970, em Botucatu-SP, após inúmeros fracassos em função da utilização de materiais muito exigentes em frio (Campo Dall'Orto et al., 1995-1998).

Nos últimos anos, a pesquisa brasileira despertou interesse em estudar o umezeiro como porta-enxerto para pessegueiros e nectarineiras, dada a proximidade parental das duas espécies e a compatibilidade da enxertia (Campo Dall'Orto et al., 1992 e 1994; Nakamura et al., 1999), sendo que também foi comprovada sua resistência aos nematóides causadores de galhas (Sherman \& Lyrene, 1983; Rossi et al., 2002; Mayer et al., 2003). Além destas vantagens, foram observadas reduções no vigor das copas em até $50 \%$, em relação às plantas enxertadas no pessegueiro cv. Okinawa (Prunus persica (L.) Batsch.), o que poderia possibilitar o adensamento dos pomares, facilitar os tratos culturais e influenciar na produtividade. O umezeiro como portaenxerto também pode promover aumentos na massa dos frutos, teor de sólidos solúveis e porcentagem de vermelho na película (Campo Dall'Orto et al., 1992 e 1994). Entretanto, nestes estudos, foram verificadas expressivas diferenças de vigor entre as plantas de uma mesma cultivarcopa, tanto no viveiro como no campo, em decorrência da propagação por sementes do umezeiro e da variabilidade genética destes.

Como tentativa de solucionar este inconveniente, diversos trabalhos com propagação por estacas herbáceas foram realizados na FCAV/UNESP, Câmpus de Jaboticabal-SP, onde foram observados resultados bastante promissores em todas as estações do ano (Nachtigal et al., 1999; Mayer et al., 2001; Mayer \& Pereira, 2002; Mayer et al., 2002; Mayer \& Pereira, 2003) e que resultaram na seleção dos Clones 05; 10 e 15 de umezeiro. Entretanto, necessita-se de informações sobre os hábitos de crescimento destes clones e as possíveis diferenças entre si e em relação à cv. Okinawa, tradicional porta-enxerto utilizado na região Sudeste brasileira.

Desta forma, o objetivo do presente trabalho foi avaliar o crescimento de três clones de umezeiro (Clones 05; 10 e 15) e do

\footnotetext{
${ }^{1}$ (Tabalho 108/2003). Recebido: 04/09/2003. Aceito para publicação: 09/02/2004. Apoio Financeiro: FAPESP. Parte da Tese de Doutorado do primeiro autor.

2 Eng. Agr., M.Sc., Aluno do Curso de Pós-Graduação em Agronomia da Faculdade de Ciências Agrárias e Veterinárias, Campus de Jaboticabal. FCAV/UNESP, Departamento de Produção Vegetal, Via de Acesso Prof. Paulo Donato Castellane Km 05, CEP 14884-900, Jaboticabal-SP. Fone: (016) 3209-2668. E-mail: namayer@fcav.unesp.br

${ }^{3}$ Eng. Agr., Dr., Prof. Titular do Departamento de Produção Vegetal da FCAV/UNESP, Câmpus de Jaboticabal-SP. E-mail: fmendes@ @cav.unesp.br
} 
TABELA 1 - Comprimento da haste (em cm) dos porta-enxertos Clones 05; 10 e 15 de umezeiro e pessegueiro cv. Okinawa, em diferentes períodos, após o transplantio das estacas enraizadas. Taquaritinga-SP, março de 2002.

\begin{tabular}{|c|c|c|c|c|c|c|}
\hline \multirow[b]{2}{*}{ Clone } & \multicolumn{6}{|c|}{ Dias após o transplante } \\
\hline & 40 & 70 & 100 & 115 & 130 & 145 \\
\hline Clone 05 & $15,48 \mathrm{c}$ & $41,90 \mathrm{c}$ & $75,05 \mathrm{~b}$ & 98,80 a & $118,87 \mathrm{a}$ & $127,60 \mathrm{a}$ \\
\hline Clone 10 & $24,30 \mathrm{ab}$ & $62,10 \mathrm{ab}$ & 93,33 a & 113,83 a & 130,83 a & $142,00 \mathrm{a}$ \\
\hline Clone 15 & $18,55 \mathrm{bc}$ & $54,40 \mathrm{~b}$ & $85,43 \mathrm{ab}$ & $103,13 \mathrm{a}$ & $116,83 \mathrm{a}$ & $130,17 \mathrm{a}$ \\
\hline Okinawa & $25,75 \mathrm{a}$ & $66,17 \mathrm{a}$ & $80,50 \mathrm{ab}$ & $82,40 \mathrm{~b}$ & $83,60 \mathrm{~b}$ & $86,93 \mathrm{~b}$ \\
\hline $\mathrm{F}$ & $8,59 * *$ & $15,63 * *$ & $4,09 * *$ & $8,96 * *$ & $16,81 * *$ & $21,13 * *$ \\
\hline $\mathrm{CV}$ & 42,90 & 26,34 & 25,16 & 24,00 & 24,04 & 23,50 \\
\hline
\end{tabular}

Médias seguidas por letras distintas, na mesma coluna, diferem entre si, pelo teste de Tukey.

** significativo ao nível de $1 \%$ de probabilidade.

pessegueiro cv. Okinawa propagados por estacas herbáceas.

\section{MATERIAISEMÉTODOS}

Para o fornecimento de material propagativo (ramos herbáceos), plantas-matrizes dos Clones 05; 10 e 15 de umezeiro (Prunus mume Sieb. et Zucc.) e do pessegueiro cv. Okinawa (Prunus persica (L.) Batsch.) foram mantidas, respectivamente, em vasos plásticos sob ripado $(50 \%$ de sombreamento) e em condições de campo, ambas as áreas pertencentes ao Departamento de Produção Vegetal da FCAV/UNESP, Câmpus de Jaboticabal-SP. Das plantas-matrizes de umezeiro, os ramos herbáceos foram coletados aos 90 dias após a poda de renovação e da cv. Okinawa, aos 60 dias. Os ramos foram acondicionados em sacos plásticos, umedecidos e transportados ao "Viveiro Paluma" (TaquaritingaSP), local onde foram realizados o enraizamento das estacas e a condução do experimento. A estaquia dos clones de umezeiro foi realizada no dia 22 de agosto de 2001 e a cv. Okinawa, 19 dias após, em função das diferenças de desenvolvimento dos ramos.

Para a propagação dos porta-enxertos, utilizaram-se estacas de $12 \mathrm{~cm}$ de comprimento com 3 a 5 folhas, tratadas com AIB a $2.000 \mathrm{mg} . \mathrm{L}^{-1}$ por cinco segundos (Mayer et al., 2001). As estacas foram acondicionadas em caixas de madeira $(48 \times 33 \times 9 \mathrm{~cm})$ contendo vermiculita fina como substrato e mantidas sob câmara de nebulização intermitente coberta com sombrite ( $25 \%$ de sombreamento).

Transcorrido o período de enraizamento, as estacas foram retiradas no dia 29 de outubro de 2001 para classificação e transplantio. As estacas enraizadas foram classificadas em uma escala de notas (que variou de 1 a 3), objetivando eliminar estacas mal enraizadas do experimento e evitar a formação de plantas com sistema radicular não satisfatório. O critério adotado para a classificação das estacas enraizadas foi visual, sendo: Nota 1= enraizamento deficiente (pequeno volume de raízes, raízes curtas e/ou inadequadamente distribuídas ao redor da base da estaca), sem condições de transplante; Nota $2=$ enraizamento satisfatório, em condições de transplantio; Nota $3=$ enraizamento excelente, em condições de transplantio.

Para o transplantio das estacas, foram utilizadas sacolas plásticas perfuradas para produção de mudas $(28 \times 18 \mathrm{~cm})$, as quais foram preenchidas até $2 / 3$ da sua capacidade com substrato (Rendmax Citrus ${ }^{\circledR}$ ), mantidas em piso cimentado e cobertas com sombrite $(50 \%$ de sombreamento). As estacas enraizadas e selecionadas foram imediatamente transplantadas após a classificação, completando-se o volume do recipiente com substrato. Foi realizada uma adubação específica para a produção de mudas (Osmocote ${ }^{\circledR}$ ), aos 19 dias após o transplantio, na dosagem de 6g/sacola plástica, de formulação 15-10-10 + micronutrientes. Foi feita também uma aplicação de calcário dolomítico ( $137 \%$ de PRNT, $45 \%$ de Cálcio e 25\% de Magnésio), na dosagem de 4 a $5 \mathrm{~g} / \mathrm{sacola}$ plástica, aos 90 dias após o transplante. As plantas foram conduzidas em haste única, sendo tutoradas com lascas de bambu com $70 \mathrm{~cm}$ de comprimento, as quais foram fixadas no substrato próximo do porta-enxerto e amarradas com barbante de algodão, quando necessário.

Para a composição do experimento, foram separadas, aleatoriamente, trinta plantas de cada clone, devidamente etiquetadas e numeradas, para a realização das avaliações de diâmetro da haste $(\mathrm{a} 5 \mathrm{~cm}$ da estaca original) e comprimento (mensurado desde sua inserção junto à estaca original até o meristema apical). As avaliações foram realizadas aos 40; 70; 100; 115; 130 e 145 dias após o transplantio das estacas para as sacolas plásticas, utilizando-se até paquímetro digital e fita métrica. $\mathrm{O}$ experimento foi conduzido em delineamento inteiramente casualizado, com 4 tratamentos (Clones 05; 10 e 15 de umezeiro e cv. Okinawa) e 30 repetições, sendo cada parcela constituída por uma planta. Os dados foram submetidos à análise de variância, pelo teste $\mathrm{F}$, e as médias foram comparadas pelo teste de Tukey, ao nível de 5\% de probabilidade. Para a análise do comprimento e diâmetro da haste principal, em função do número de dias após o transplantio, utilizou-se a regressão polinomial até $3^{\circ}$ grau, de acordo com Gomes (1966).

\section{RESULTADOSE DISCUSSÃO}

Os valores referentes ao comprimento da haste são apresentados na Tabela 1. Verifica-se que, aos 40 e 70 dias após o transplante, a cv. Okinawa apresentou maior crescimento em relação aos Clones 05 e 15, sendo que não diferiu estatisticamente do Clone 10. Já aos 100 dias, o Clone 15 igualou-se estatisticamente ao Clone 10 e ao 'Okinawa'. A partir desta avaliação, a cv. Okinawa estabilizou o crescimento. Entretanto, para os três clones de umezeiro, o crescimento continuou constante (Figura 1), resultando em plantas mais altas, com maiores comprimentos de haste e que não diferiram entre si, até o final das avaliações. Para os três clones de umezeiro estudados, a regressão linear foi a que melhor se ajustou às médias obtidas, sendo que para a cv. Okinawa foi a regressão quadrática (Figura 1).

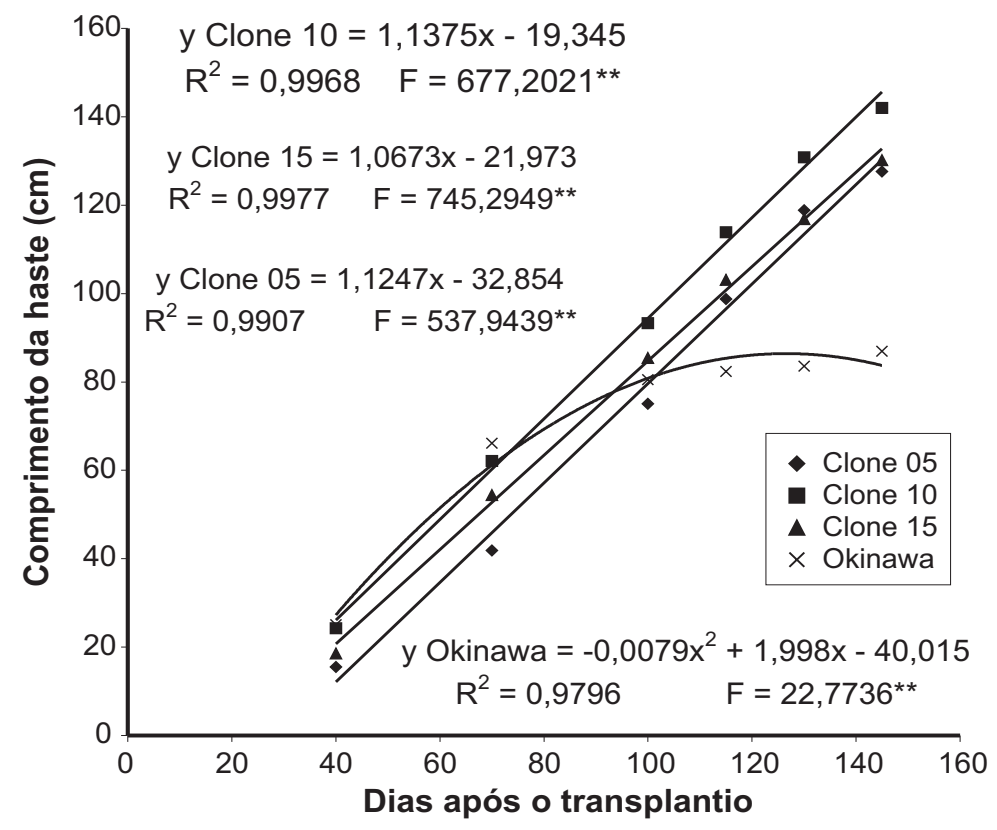

FIGURA 1 - Comprimento da haste de clones de umezeiro e pessegueiro cv. Okinawa propagados por estacas herbáceas, nas diferentes avaliações, após o transplantio para sacolas plásticas. 
TABELA 2 - Diâmetro da haste a $5 \mathrm{~cm}$ da estaca original $(\mathrm{em} \mathrm{mm})$ dos porta-enxertos Clones $05 ; 10$ e 15 de umezeiro e pessegueiro cv. Okinawa, em diferentes períodos, após o transplantio das estacas enraizadas. Taquaritinga-SP, março de 2002.

\begin{tabular}{ccccccc}
\hline & \multicolumn{7}{c}{ Dias após o transplante } \\
\cline { 2 - 7 } Clone & 40 & 70 & 100 & 115 & 130 & 145 \\
\hline Clone 05 & $1,86 \mathrm{~b}$ & $2,45 \mathrm{c}$ & $3,16 \mathrm{~b}$ & $3,55 \mathrm{~b}$ & $3,94 \mathrm{~b}$ & $4,65 \mathrm{~b}$ \\
Clone 10 & $2,54 \mathrm{a}$ & $3,14 \mathrm{~b}$ & $3,67 \mathrm{~b}$ & $3,96 \mathrm{~b}$ & $4,42 \mathrm{~b}$ & $5,09 \mathrm{ab}$ \\
Clone 15 & $2,08 \mathrm{~b}$ & $2,76 \mathrm{bc}$ & $3,33 \mathrm{~b}$ & $3,58 \mathrm{~b}$ & $3,85 \mathrm{~b}$ & $4,61 \mathrm{~b}$ \\
Okinawa & $2,70 \mathrm{a}$ & $4,09 \mathrm{a}$ & $5,13 \mathrm{a}$ & $5,38 \mathrm{a}$ & $5,67 \mathrm{a}$ & $6,09 \mathrm{a}$ \\
\hline $\mathrm{F}$ & $11,75^{* *}$ & $23,82^{* *}$ & $19,11^{* *}$ & $13,83^{* *}$ & $10,78^{* *}$ & $3,74^{* *}$ \\
\hline CV & 26,95 & 25,68 & 29,42 & 30,92 & 31,28 & 30,84 \\
\hline
\end{tabular}

Médias seguidas por letras distintas, na mesma coluna, diferem entre si, pelo teste de Tukey.

** significativo ao nível de $1 \%$ de probabilidade.

Os valores observados para a variável diâmetro da haste são apresentados na Tabela 2. Verifica-se que, aos 40 dias após o transplante das estacas para as sacolas plásticas, o diâmetro (a $5 \mathrm{~cm}$ da estaca original) da cv. Okinawa foi estatisticamente semelhante ao observado no Clone 10; entretanto, ambos foram superiores aos Clones 05 e 15. Nas avaliações seguintes, até os 130 dias, a cv. Okinawa apresentou maior diâmetro em relação aos três clones de umezeiro estudados. Dentre os clones de umezeiro, as diferenças estatísticas só foram observadas aos 40 e 70 dias após o transplantio. Nesta variável, todos os porta-enxertos ajustaram-se à regressão linear, conforme ilustra a Figura 2. Aos 130 dias, aproximadamente $33 \%$ dos porta-enxertos de umezeiro encontraramse aptos à realização da enxertia, segundo observações visuais.

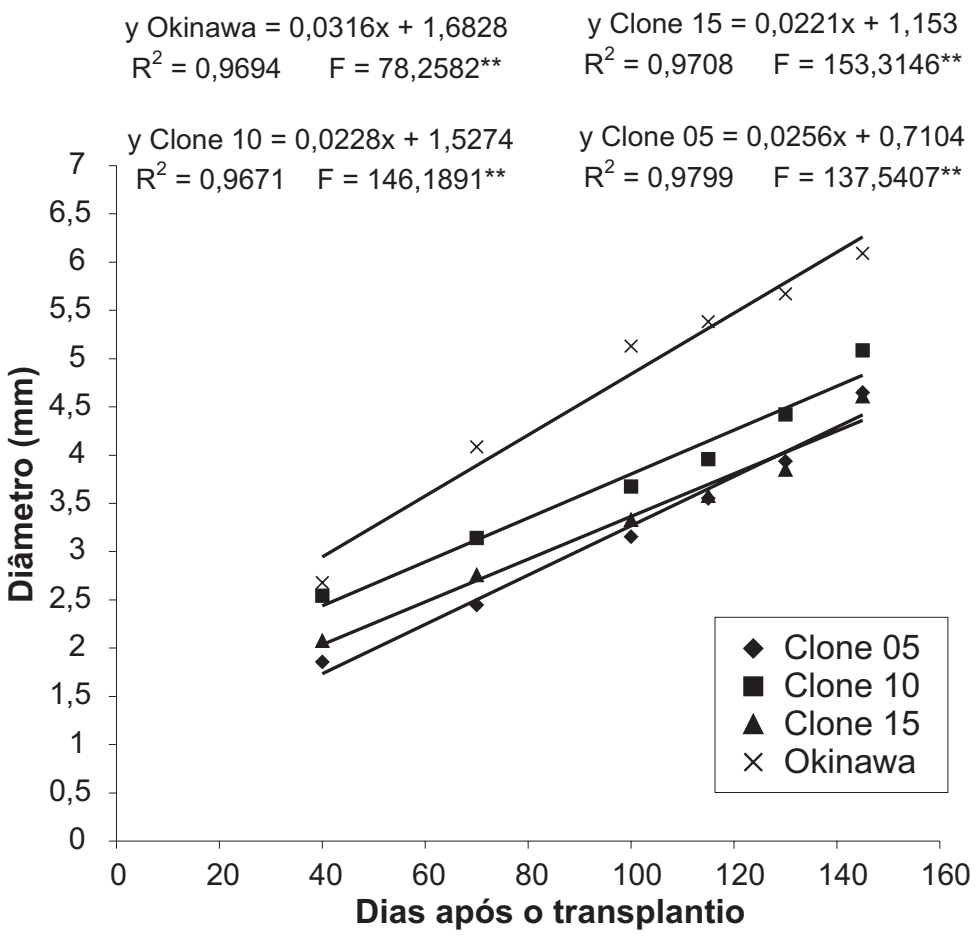

FIGURA 2: Diâmetro da haste a $5 \mathrm{~cm}$ da estaca original de clones de umezeiro e cv. Okinawa propagados por estacas herbáceas, nas diferentes avaliações, após o transplantio para sacolas plásticas.

Estes dados, juntamente com os de comprimento da haste, demonstram claramente os diferentes hábitos de crescimento das duas espécies. O umezeiro apresenta intenso crescimento da haste em comprimento, com diâmetro relativamente homogêneo ao longo da sua extensão, caracterizando um crescimento contínuo. Já o 'Okinawa' apresenta maior diâmetro na base da haste (a $5 \mathrm{~cm}$ da estaca original) em relação aos clones de umezeiro, rápido crescimento inicial da haste até os 100 dias e posterior estabilização, refletindo-se em plantas mais baixas (Figura 1), porém com maior porcentagem de plantas aptas à realização da enxertia (em torno de 55\%), em função do maior diâmetro. Desta forma, em termos práticos, pode-se destacar que, no caso do umezeiro, o comprimento da haste, que define a altura do porta-enxerto, não é uma característica importante para a definição da porcentagem de plantas aptas à enxertia, em função da característica de crescimento contínuo.

Verifica-se, com estas observações, que a propagação de portaenxertos de umezeiro e 'Okinawa' por estacas herbáceas pode apresentar resultados satisfatórios e viáveis tecnicamente. Salienta-se, entretanto, que não foram encontrados na literatura trabalhos que comparassem os hábitos de crescimento de umezeiro com a cv. Okinawa propagados por estacas herbáceas, para fins de posterior utilização como porta-enxertos para pessegueiro. Campo Dall'Orto et al. (1992) apenas relatam que portaenxertos da seleção de umezeiro 'Iacume' (IAC-3), obtidos por germinação de amêndoas em sacolas plásticas, foram levados para o campo com doze meses de idade e enxertados oito dias após o transplantio. Nesta ocasião, os porta-enxertos selecionados apresentavam, aproximadamente, $1 \mathrm{~m}$ de altura e $1 \mathrm{~cm}$ de diâmetro basal.

\section{CONCLUSÕES}

Nas condições em que o experimento foi conduzido, pode-se concluir que:

1) Os Clones 05; 10 e 15 de umezeiro apresentam crescimento contínuo com relação ao comprimento da haste, enquanto a cv. Okinawa estabiliza o crescimento após 100 dias do transplantio, refletindo-se em plantas mais baixas.

2) Não existem diferenças entre os clones de umezeiro estudados no diâmetro da haste após 100 dias e no comprimento da haste após 115 dias do transplantio das estacas enraizadas.

3) A cv. Okinawa apresenta maior diâmetro da haste em relação aos Clones 05; 10 e 15 de umezeiro, o que define maior porcentagem de porta-enxertos aptos à realização da enxertia.

\section{AGRADECIMENTOS}

À Fundação de Amparo à Pesquisa do Estado de São Paulo, pela concessão da bolsa de estudos e suporte financeiro para a execução deste trabalho.

Os autores agradecem a colaboração dos Srs. José Mauro da Silva e João Matheus da Silva, proprietários do "Viveiro Paluma" (Taquaritinga-SP), local onde o experimento foi conduzido.

\section{REFERÊNCIASBIBLIOGRÁFICAS}

CAMPO DALL'ORTO, F.A.; OJIMA, M.; BARBOSA, W.; MARTINS, F.P. O nanismo do pessegueiro induzido pela enxertia no damasqueiro-japonês. Pesquisa Agropecuária Brasileira, Brasília, v.27, n.3, p.517-521, 1992.

CAMPO DALL'ORTO, F.A.; BARBOSA, W.; OJIMA, M.; MARTINS, F.P.; FOBÉ, L.A. Comportamento de pessegueiros IAC enxertados no damasqueiro-japonês e no pessegueiro 'Okinawa'. In: CONGRESSO BRASILEIRO DE FRUTICULTURA, 13., 1994, Salvador. Anais... Salvador: SBF, v. 3, 1994. p.879-880.

CAMPO DALL'ORTO, F.A.; OJIMA, M.; BARBOSA, W.; MARTINS, 
F.P. Damasco-japonês (umê) em São Paulo: opção para o século 21. O Agronômico, Campinas, v.47/50, p.18-20, 1995/1998. (Boletim Técnico Informativo).

GOMES, F.P. Curso de estatística experimental. 3. ed. Piracicaba: ESALQ, 1966. 436p.

MAYER, N.A.; PEREIRA, F.M.; NACHTIGAL, J.C. Propagação do umezeiro (Prunus mume Sieb \& Zucc.) por estaquia herbácea. Revista Brasileira de Fruticultura, Jaboticabal, v.23, n.03, p.673-676, 2001.

MAYER, N.A.; PEREIRA, F.M. Incision effect in the cuttings herbaceous base in the rooting of four japanese apricot clones (Prunus mume Sieb. et Zucc.) in intermittent mist. In: INTERNATIONAL SYMPOSIUMONROOTSTOCKSFOR DECIDUOUS FRUIT TREE SPECIES, 1., 2002, Zaragoza. Abstracts... Zaragoza: ISHS, CSIC, CIHEAM, 2002. p.13.

MAYER, N.A.; PEREIRA, F.M.; NACHTIGAL, J.C. Efeito do comprimento de estacas herbáceas de dois clones de umezeiro (Prunus mume Sieb \& Zucc.) no enraizamento adventício. Revista Brasileira de Fruticultura, Jaboticabal, v.24, n.02, p.500-504, 2002.

MAYER, N.A.; PEREIRA, F.M. Enraizamento de estacas herbáceas de quatro clones de umezeiro (Prunus mume Sieb. et Zucc.) durante o inverno ameno, em Jaboticabal-SP. Revista Brasileira de Fruticultura, Jaboticabal, v.25, n.03, p.505-507, 2003.
MAYER, N.A.; PEREIRA, F.M.; SANTOS, J.M. dos. Reação de clones de umezeiro (Prunus mume Sieb. et Zucc.) e cultivares de pessegueiro a Meloidogyne javanica (Treub, 1885) Chitwood, 1949. Revista Brasileira de Fruticultura, Jaboticabal, v.25, n.01, p.181$183,2003$.

NACHTIGAL, J.C.; PEREIRA, F.M.; CAMPODALL'ORTO, F.A.; OJIMA, M.; MARTINS, F.P. Propagação vegetativa do umezeiro (Prunus mume) por meio de estacas herbáceas. Revista Brasileira de Fruticultura, Jaboticabal, v.21, n.2, p.226-228, 1999.

NAKAMURA, C.H.; SCARPARE FILHO, J.A.; KLUGE, R.A. Avaliação preliminar do umezeiro como porta-enxerto para pessegueiro e nectarineira. Revista Brasileira de Fruticultura, Jaboticabal, v.21, n.2, p.116-118, 1999.

ROSSI, C.E.; FERRAZ, L.C.C.B.; MONTALDI, P.T. Resistência de frutíferas de clima subtropical e temperado a Meloidogyne incognita raça 2 e M.javanica. Arquivos do Instituto Biológico, São Paulo, v.69, n.2, p.43-49, 2002.

SHERMAN, W.B.; LYRENE, P.M. Improvement of peach rootstock resistant to root-knot nematodes. Proceedings of the Florida State Horticultural Society, Winter Haven, v. 96, p.207-208, 1983.

YOSHIDA, M. Mume, plum and cherry. In: Horticulture in Japan. Tokyo: Chuo Printing, 1994. p. 37-38. 